\title{
Article \\ Characterization of Salmonella Isolates Recovered from Stages of the Processing Lines at Four Broiler Processing Plants in Trinidad and Tobago
}

\author{
Anisa Sarah Khan ${ }^{1}$, Karla Georges ${ }^{1}$ (D), Saed Rahaman ${ }^{2}$, Woubit Abebe ${ }^{3}$ and Abiodun Adewale Adesiyun ${ }^{1,4, *(D)}$ \\ 1 School of Veterinary Medicine, Faculty of Medical Sciences, University of the West Indies, St. Augustine, \\ Trinidad and Tobago; anisakhan11@gmail.com (A.S.K.); Karla.georges@sta.uwi.edu (K.G.) \\ 2 Veterinary Public Health Unit, Ministry of Health, Port of Spain, Trinidad and Tobago; \\ saed.rahaman@gmail.com \\ 3 Department of Pathobiology, Tuskegee University College of Veterinary Medicine, Tuskegee, \\ 201 Frederick D Patterson Dr, Tuskegee, AL 36088, USA; wabdela@tuskegee.edu \\ 4 Department of Production Animal Studies, Faculty of Veterinary Science, University of Pretoria, \\ Private Bag X04, Onderstepoort, Pretoria 0110, South Africa \\ * Correspondence: abiodun.adesiyun@sta.uwi.edu
}

\section{check for}

updates

Citation: Khan, A.S.; Georges, K.; Rahaman, S.; Abebe, W.; Adesiyun, A.A. Characterization of Salmonella Isolates Recovered from Stages of the Processing Lines at Four Broiler Processing Plants in Trinidad and Tobago. Microorganisms 2021, 9, 1048. https://doi.org/10.3390/ microorganisms 9051048

Academic Editors: María-Jesús Grilló and Lourdes Migura-Garcia

Received: 12 April 2021

Accepted: 10 May 2021

Published: 13 May 2021

Publisher's Note: MDPI stays neutral with regard to jurisdictional claims in published maps and institutional affiliations.

Copyright: (c) 2021 by the authors. Licensee MDPI, Basel, Switzerland. This article is an open access article distributed under the terms and conditions of the Creative Commons Attribution (CC BY) license (https:// creativecommons.org/licenses/by/ $4.0 /)$.

\begin{abstract}
This cross-sectional study determined the prevalence, characteristics, and risk factors for contamination of chicken with Salmonella at four operating broiler processing plants in Trinidad. Standard methods were used to isolate and characterize the Salmonella isolates. The overall prevalence of Salmonella at the four processing plants was 27.0\% (107/396). The whole carcass enrichment (WCE) method yielded a statistically significantly $(p=0.0014)$ higher frequency of isolation $(53.9 \% ; 97 / 180)$ than the whole carcass rinse $(35.0 \% ; 63 / 180)$ and neck skin methods $(42.2 \% ; 38 / 90)$. S. enterica serotypes Enteritidis, Javiana, and Infantis were the predominant serotypes isolated accounting for $20.8 \%, 16.7 \%$ and $12.5 \%$, respectively, of the serotyped isolates. Risk factors included the use of over 100 contract farmers (OR 4.4), pre-chiller (OR 2.3), addition of chlorine to chiller (OR 3.2), slaughtering sick broilers (OR 4.4), and flocks with $>50 \%$ mortality. Multi-drug resistance was detected in $12.3 \%(14 / 114)$ of the isolates of Salmonella. Resistance was high to kanamycin (85.7\%) and doxycycline $(74.6 \%)$ but low to amoxicillin-clavulanic acid $(2.4 \%)$ and sulphamethoxazoletrimethoprim $(0.8 \%)$. The occurrence of resistant Salmonella in chickens processed at commercial broiler processing plants has implications for salmonellosis and therapeutic failure in consumers of improperly cooked contaminated chickens from these plants in the country.
\end{abstract}

Keywords: broiler processing plants; Salmonella; serotypes; risk factors; antimicrobial resistance; Trinidad

\section{Introduction}

Salmonellosis is the third leading cause of death among food transmitted diseases [1] with an estimated global Salmonella enterocolitis incidence of 95.1 million cases [2], accounting for 50,771 deaths in 2017 [3]. In the Caribbean, Salmonella is the most common laboratory-confirmed cause of foodborne diseases since 2005 [4]. Poultry has been reported to be the main carrier of Salmonella infections to humans [5], more common than any other animal species [6]. Broiler meat is an economical source of protein and estimated to be the most widely consumed meat, globally.

The human population of the twin-island Republic of Trinidad and Tobago is 1,366,725 [7] with a reported $58.3 \mathrm{~kg}$ per capita poultry consumption rate; 800,000 broilers are produced weekly, of which $20 \%$ are imported [8]. Consumers purchase chicken from cottage poultry processors, where they are freshly slaughtered and from supermarkets, which offer both chilled and frozen locally processed or imported frozen chicken. Broiler processing plants are responsible for $50 \%$ of local broiler processing [9] where supermarkets and the fran- 
chised foodservice sector are supplied with chilled chickens as well as further-processed products [9].

Several studies have reported the high frequency of contamination with Salmonella of chicken meat sold at the informal and formal outlets in developed and developing countries $[10,11]$. It has also been reported that the processing of chicken at commercial processing plants contributes significantly to the contamination of dressed chicken carcasses with Salmonella before they reach the retail outlets [12,13]. Unhygienic carcass handling, soiled slaughter equipment [14,15], contaminated water (scalding and immersion chiller water), and waste generated from evisceration and the de-feathering processes have been implicated as major sources of Salmonella contamination during broiler processing [16-18]. Salmonella-free broilers leaving farms may potentially become contaminated by the pathogen during processing through contact with immersion chiller water contaminated with Salmonella originating from the positive broilers $[19,20]$. This can occur, should there be improper $\mathrm{pH}$ and chemical agents' concentrations, as well as a failure to maintain good sanitary practices throughout processing [21].

With the increase in production and consumption of broiler meat over the years, the use, misuse, and overuse of veterinary drugs for prophylaxis, therapeutic, and growth promotion purposes $[22,23]$ are common in countries such as Trinidad and Tobago. In the country, although regulations on the use of veterinary drugs in livestock exist, they are not routinely enforced. The increase in the isolation of Salmonella in humans, and the resistance of Salmonella strains to antimicrobial agents commonly used in food-producing animals is a major health concern $[24,25]$. Worldwide, of a greater concern is the emergence of multidrug-resistant (MDR) Salmonella [26], which has been implicated in foodborne outbreaks due to contaminated meat $[27,28]$.

To isolate Salmonella from poultry processing plants, different approaches have been reported and recommended. In the European Union, the use of neck skin (NS) maceration [29] is most frequent whereas, in the United States, the U.S. Department of Agriculture Food Safety and Inspection Service (USDA-FSIS) [30] recommends the use of whole carcass rinse (WCR) method. Whilst the WCR is the most commonly used method for isolation of Salmonella in broiler carcasses [31-33], the whole carcass enrichment (WCE) and neck skin (NS) methods have been shown to be just as effective [34] or even more than the WCR [35]. However, the large space required for incubating whole carcasses makes the WCE method impractical for routine testing, but it is valuable for research purposes [36].

In Trinidad and Tobago and the Caribbean, there is a dearth of comprehensive up-todate data on the role played by the commercial broiler processing plants in the contamination of processed chicken carcasses with Salmonella. The only available recent published data were from studies conducted at the outlets of cottage poultry processors ('wet market') where the slaughtering and retailing of dressed chicken were practiced [37] and at supermarkets where retailing of chicken from the commercial processing plants occurs [38] and the antimicrobial resistance profiles of Salmonella isolates from both sources were determined [39].

Considering the limited current information on the status and dynamics of Salmonella contamination of chicken carcasses at the commercial broiler processing plants, the present study with the following objectives was conducted: (i) to determine the frequency of isolation of Salmonella longitudinally from the different stages of processing, from preslaughter broilers to chilled carcasses, (ii) to evaluate the efficacy of three isolation methods for Salmonella, (iii) to identify the risk factors associated with Salmonella contamination of chicken carcasses at the plants and finally, (iv) to determine the serotypes and antimicrobial resistance profiles of the isolates of the pathogen recovered from the four plants operating in Trinidad. 


\section{Materials and Methods}

\subsection{Sampling Site}

The study was conducted in Trinidad and Tobago, the twin-island Caribbean country located in the southern Caribbean, north-east of the South American country of Venezuela, northwest of Guyana, and south of Grenada in the Lesser Antilles. There are currently four commercial broiler processing plants in Trinidad. These plants process only broiler chickens and supply supermarkets and food outlets with dressed chilled and/or frozen chicken. Each processing plant packages whole dressed chickens, various packaged chicken parts (legs, thighs, breasts, wings, and mixed parts), offal (liver, gizzard), feet, and necks all of which are available for sale at their retail outlet (at the respective plant) or supplied to supermarkets or food outlets. The similarities and differences in the operations that may impact on the bacteriological quality of broilers at the four processing plants studied are shown in a flow chart (Supplementary data: S1, A-D).

The number of samples to be collected for this study was estimated using the formula [40]: Estimated sample size for an infinite population,

$$
n_{o}=\mathrm{Z}_{\mathrm{u}}^{2} \mathrm{P}_{\mathrm{ex}}\left(1-\mathrm{P}_{\mathrm{ex}}\right) / \mathrm{d}^{2}
$$

where:

$n_{0}=$ Estimated sample size; $Z_{\mathrm{u}}=$ Degree of confidence $=1.96 ;$

$\mathrm{P}_{\mathrm{ex}}=$ Expected prevalence $=50 \% ; \mathrm{d}=$ Desired absolute precision $=5 \%$;

$n_{0}=\left[1.96^{2} \times 0.5(1-0.5)\right] / 0.05^{2}=384$.

A total of 396 samples were collected comprising swabs of pre-slaughter cloacae, preevisceration carcasses, post-evisceration carcasses, chilled whole chickens (dressed), and chilled chicken parts (dressed), as well as neck skins and chiller water. Sample collection was conducted during the period from January to September 2019. The total number of samples collected at each plant was determined using proportional sampling based on their throughputs. Therefore, two, four, one, and two sampling visits were made to plants A, B, C, and D, respectively. Plant A and D received chicken from their 210 and 98 contract farms, respectively, whereas Plants $B$ and $C$ were owned by the same parent company that controlled 32 farms. Samples were collected in individual sterile bags and bottles and transported on ice to the laboratory of the Veterinary Public Health Unit, School of Veterinary Medicine for processing within 4-6 h after collection. Standardized, pre-tested questionnaires were administered at each broiler processing plant to obtain information about demography, operational information, and risk factors for carcass contamination with Salmonella. Some of the questions were designed to elicit information on the average number of contract farmers, the average waiting period between arrival of chickens to slaughter, disposal of waste material, and source of water supply (Supplementary data: S2).

\subsection{Processing of Samples Collected from Processing Plants}

During each visit to the broiler processing plant the following samples were collected in sterile bottles/bags: 10 cloacal swabs, 5 pre-evisceration carcasses (post-defeathering), 5 post-evisceration carcasses, 10 neck skins, 4 immersion chiller water samples, 5 chilled whole carcasses (after removal from immersion chiller), and 5 packs of chilled chicken parts each of legs, thighs, breast, wings, and mixed parts.

The WCR method, described by the USDA-FSIS [30] for Salmonella isolation was used. Each carcass was rinsed in $430 \mathrm{~mL}$ of buffered peptone water (BPW) (Oxoid, Hampshire, UK), rotated for no less than 30 times and $30 \mathrm{~mL}$ of the rinsate was removed and incubated.

Each carcass with the remaining $400 \mathrm{~mL}$ BPW in the WCR process above, was incubated in accordance with the WCE method as described by Cox et al. [41] and constituted the WCE sample. Neck skin (NS) samples were processed as recommended by the Commission Regulation (EC) No 2073/2005 [42] with the following modification. Each neck skin was collected in a sterile bag from which approximately 10-15 g was aseptically excised 
and added to BPW in a 1:9 ratio and incubated at $37^{\circ} \mathrm{C}$ for $18-24 \mathrm{~h}$. Each excised neck skin was treated as one (1) sample as performed in other studies [34,43].

Each cloacal swab sample was added to $9 \mathrm{~mL}$ BPW and subsequently incubated [44]. During each sampling visit to the plants, $400 \mathrm{~mL}$ of immersion chiller water was collected four (4) times, at an interval of $1.5 \mathrm{~h}$ to provide representative samples of potential contamination over a $6 \mathrm{~h}$ period. In the laboratory, $100 \mathrm{~mL}$ were aseptically removed from each $400 \mathrm{~mL}$ sample and centrifuged at $4470 \times g$ for 20 min after which $1 \mathrm{~mL}$ of sediment was removed and transferred to $9 \mathrm{~mL} \mathrm{BPW}$ and incubated [45].

All pre-enriched BPW samples were incubated at $37^{\circ} \mathrm{C}$ for $18-24 \mathrm{~h}$. Samples were then selectively enriched in $9 \mathrm{~mL}$ tetrathionate (TT) broth (Oxoid, Hampshire, UK) and $9 \mathrm{~mL}$ Rappaport-Vassiliadis Soya (RVS) broth (Oxoid, Hampshire, UK) and incubated at 37 and $42{ }^{\circ} \mathrm{C}$, respectively.

\subsection{Isolation and Identification of Salmonella}

Samples enriched in selective broths were sub-cultured onto Xylose-lysine-tergitol 4 (XLT-4; Oxoid, Hampshire, UK) and Brilliant green agar (BGA; Oxoid) and incubated at $37^{\circ} \mathrm{C}$ for $18-24 \mathrm{~h}$. Suspected Salmonella colonies that displayed characteristic colonies on both selective agar plates were then purified on blood agar plates (Oxoid) and incubated at $37^{\circ} \mathrm{C}$ for $18-24 \mathrm{~h}$. Pure cultures were subjected to a panel of biochemical tests that included triple sugar iron agar, lysine iron agar, urea, citrate, methyl red, sulfide-indole-motility medium, and o-nitrophenyl-b-D-galactopyranoside (Oxoid) [39,46]. Isolates biochemically confirmed as Salmonella were then subjected to a slide agglutination test using Salmonella polyvalent antiserum (A-I \& Vi, Difco, Detroit, MI). Complete confirmation and serotyping of Salmonella isolates representative of those recovered by the WCR/WCE/NS, RVS/TT, and BGA/XLT-4 methods were performed using the phase reversal technique, and the results interpreted according to the Kauffman-White scheme [47] at the Public Health Laboratory, Ministry of Health, St. Michael, Barbados. Molecular confirmation of tentatively identified Salmonella was conducted using conventional polymerase chain reaction (PCR). Initially, DNA was extracted from the Salmonella isolates by the boiling method $[37,48]$, followed by the use of conventional PCR to detect the invA gene as described earlier [37,48]. The following primer sequences were used to amplify a $284 \mathrm{bp}$ fragment of the invA gene, Forward: 5' GTGAAATTATCGCCACGTTCGGGCAA $3^{\prime}$ and Reverse: $5^{\prime}$ TCATCGCACCGTCAAAGGAACC $3^{\prime}$ as described by Oliviera et al. [49].

\subsection{Determination of Antimicrobial Resistance}

The antimicrobial resistance of 126 Salmonella isolates recovered from the samples obtained at the four broiler processing plants was determined using the disk diffusion method according to the Clinical and Laboratory Standards Institute $[50,51]$ guidelines. Eight antimicrobial agents commonly available in the local market and frequently used in the poultry industry in Trinidad formed the panel of antimicrobial agents. The antimicrobial agents, concentrations, and classes (Difco, Becton Dickinson, Sparks, MD, USA) used comprised the following: amoxicillin-clavulanic acid (AMC, $30 \mu \mathrm{g}, \beta$-lactam); doxycycline (DO, $30 \mu \mathrm{g}$, Tetracycline); ceftriaxone (CRO, $30 \mu \mathrm{g}$, Cephalosporin); gentamicin (CN, $10 \mu \mathrm{g}$, Aminoglycosides); kanamycin (K, $30 \mu \mathrm{g}$, Aminoglycosides); chloramphenicol (C, $30 \mu \mathrm{g}$, Phenicol); sulphamethoxazole-trimethoprim (SXT, 23.75 and $1.25 \mu \mathrm{g}$, Sulphonamides); and ciprofloxacin (CIP, $5 \mu \mathrm{g}$, Fluoroquinolones). The tests were performed on Mueller-Hinton agar (Difco), followed by aerobic incubation at $37^{\circ} \mathrm{C}$ for $24 \mathrm{~h}$. The zones of inhibition were interpreted as recommended by the disk manufacturer and Clinical and Laboratory Standards Institute [50].

\subsection{Statistical Analysis of Data}

Chi-square analyses were conducted using the Statistical Package for Social Sciences, SPSS (version 27, IBM Corp., Somers, NY, USA) to determine statistically significant associations in the frequency of isolation of Salmonella amongst (i) the three different 
sampling methods, (ii) the risk factors associated with Salmonella contamination, (iii) the types of samples collected, and iv) the plants sampled. The Fisher's exact test was used for $2 \times 2$ contingency tables with expected frequencies of $<5$. The level of significance was set at an alpha $=0.05$. Univariate analysis of associations was conducted using the Salmonella status of the sample as a binary outcome (positive or negative). The predictor variables were the average number of farmers, number of workers directly involved in processing, waiting period, the mortality rate on arrival, treatment of diseased birds, use of a pre-chiller, agents used in chiller, temperature of chiller water, and segregation of workers. Each predictor variable was tested for significant associations with the Salmonella status using the chi-square test of association. Significant variables $(p<0.05)$ in the univariate analysis were assessed for collinearity using the chi-square statistic and were considered collinear if $p<0.05$. A forward stepwise regression model where entry of $p<0.5$ and removal of $p<0.10$ was used in the regression analysis. Hosmer-Lemeshow chi-square was used as a goodness of fit test. Statistical analysis was done using SPSS (version 27) at an alpha level of 0.05 .

\section{Results}

\subsection{Overview of Management and Production Data}

In Trinidad, the poultry industry is vertically integrated, where each company controls its respective hatcheries, contracted farms, feed mills, and processing plant. However, because of the limited supply of broilers to the smaller integrated companies, broilers often originated from competitor farms. A summary of the management and production data on the four processing plants is shown in Table 1.

Table 1. Management and production data from four broiler processing plants in Trinidad.

\begin{tabular}{|c|c|c|c|c|}
\hline \multirow{2}{*}{ Parameter } & \multicolumn{4}{|c|}{ Processing Plant: } \\
\hline & Plant A & Plant B & Plant C & Plant D \\
\hline $\begin{array}{l}\text { Total installed capacity of the processing } \\
\text { plant (birds/week) }\end{array}$ & 160,000 & 250,000 & $<100,000$ & 100,000 \\
\hline Average number of broilers processed daily & 32,000 & 50,000 & 15,000 & 20,000 \\
\hline Number of days operational weekly & 5 & 5 & 4 & 5 \\
\hline Average number of contract farmers used & 210 & 32 & 32 & 100 \\
\hline $\begin{array}{c}\text { Number of workers directly involved in } \\
\text { Processing a }\end{array}$ & 150 & 400 & 75 & 150 \\
\hline $\begin{array}{c}\text { Number of workers indirectly involved in } \\
\text { Processing } b\end{array}$ & 100 & 1000 & 1000 & 75 \\
\hline $\begin{array}{l}\text { Waiting period }(\mathrm{h}) \text { between arrival of birds at } \\
\text { plant and slaughter }\end{array}$ & $2-6$ & $0.5-3$ & $1-3$ & 12 \\
\hline $\begin{array}{c}\text { Average mortalities (\%) or broilers dead on } \\
\text { arrival at plant }\end{array}$ & 0.7 & 0.02 & 0.94 & 0.50 \\
\hline $\begin{array}{l}\text { Disposal of solid waste (fecal materials) from } \\
\text { broilers }\end{array}$ & Rendered $^{\mathrm{c}}$ & External Company & Rendered & Rendered \\
\hline Disposal of waste-water & River & Settling ponds & Settling ponds & Settling ponds \\
\hline Treatment of water at the plant ${ }^{d}$ & No & Yes & No & No \\
\hline
\end{tabular}

a Workers who have contact with the birds/carcass at one point during processing; ${ }^{b}$ Workers involved in the management of the plant but not having contact with the birds/carcass during processing; ${ }^{c}$ Rendering (in-house) to convert animal tissue waste to useable by-product meal; ${ }^{\mathrm{d}}$ All plants utilized municipal water supply as their source. 


\subsection{Comparison of Sampling Methods}

Salmonella was isolated from $35.0 \%, 53.9 \%$, and $42.2 \%$ of samples subjected to the WCR, WCE, and neck skin methods, respectively $(p=0.0013)$ (Figure 1). Significant differences in the frequency of isolation of Salmonella by sampling method were found in pre-evisceration carcasses $(p<0.001)$, post-evisceration carcasses $(p<0.001)$, and all samples $(p<0.001)$. Chilled whole carcasses subjected to the WCE method yielded a higher frequency of isolation $(60 \% ; 27 / 45)$ when compared to the WCR method $(31.1 \% ; 14 / 45)$ $(p=0.01)$. Selective enrichment in tetrathionate broth plated onto XLT-4 agar yielded the highest frequency of Salmonella positive samples among the three methods $(p<0.001)$. Overall, $8.9 \%(40 / 450), 29.8 \%(134 / 450), 1.8 \%(8 / 450)$, and $3.6 \%(16 / 450)$ of the samples were isolated on RVS/XLT-4, TT/XLT-4, RVS/BGA, and TT/BGA, respectively $(p<0.001)$.

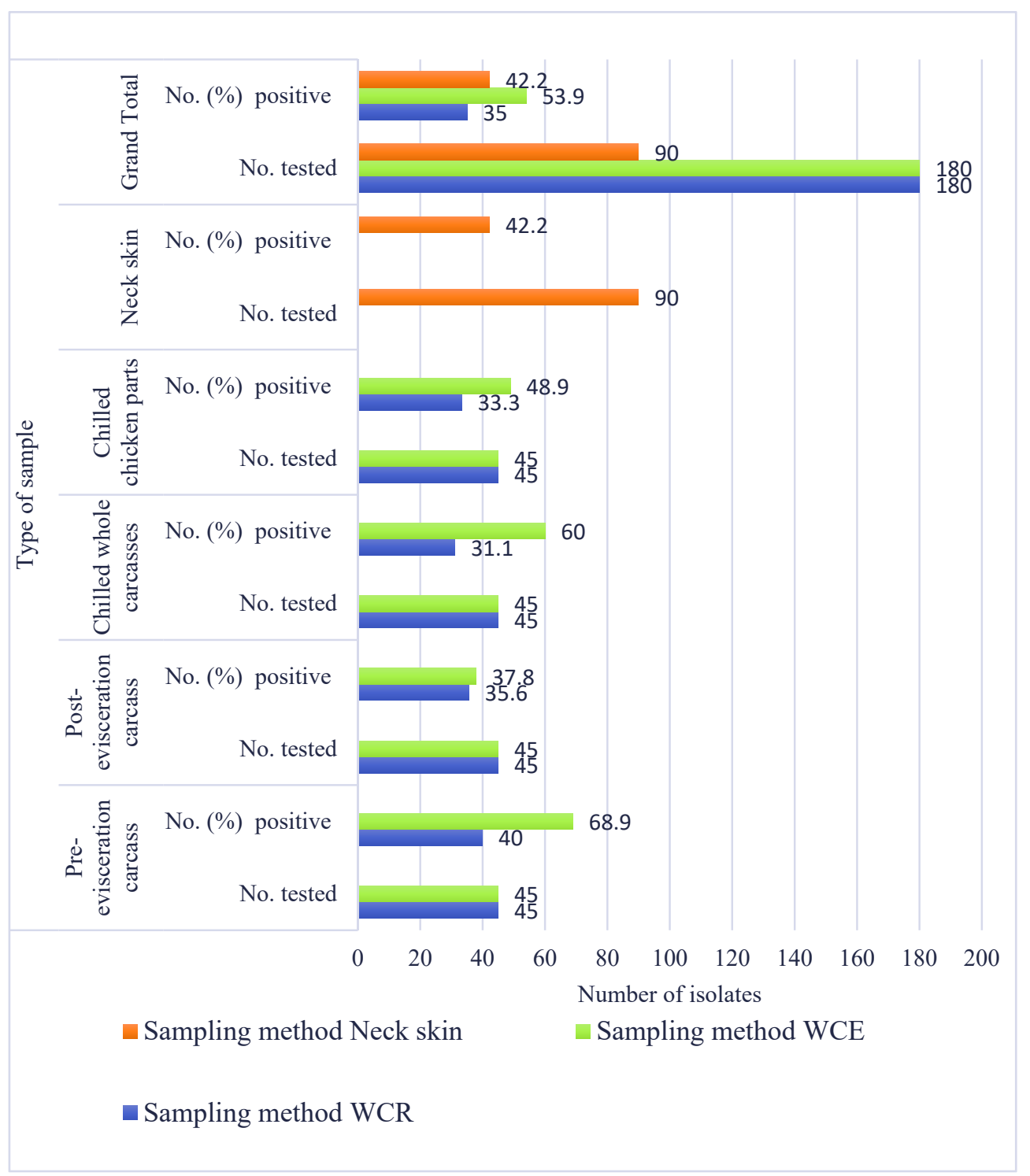

Figure 1. Recovery of Salmonella based on the method used.

\subsection{Risk Factors Associated with Salmonella Contamination during Broiler Processing}

The association of risk factors with the frequency of contamination of chickens processed is shown in Table 2. Of the 14 risk factors investigated, 10 (71.4\%) were determined to be statistically significantly associated with the contamination with Salmonella during processing. 
Table 2. Risk factors associated with Salmonella contamination of carcasses.

\begin{tabular}{|c|c|c|c|c|c|}
\hline Risk Factor & $\begin{array}{c}\text { Total No. } \\
\text { Samples Tested }\end{array}$ & $\begin{array}{l}\text { Total No. (\%) Positive } \\
\quad \text { for Salmonella }\end{array}$ & $p$-Value & Odds Ratio & CI $(95 \%)$ \\
\hline \multicolumn{3}{|l|}{ Size of plant ${ }^{\mathrm{a}}$} & $p<0.001$ & & \\
\hline Small & 44 & $5(11.4)$ & & Ref & \\
\hline Medium & 176 & $70(39.8)$ & & 5.1 & $1.94-13.71$ \\
\hline Large & 176 & $32(18.2)$ & & 1.7 & $0.63-4.74$ \\
\hline \multicolumn{3}{|c|}{ Average number of contract farmers } & $p<0.001$ & & \\
\hline$\leq 100$ farmers & 308 & $61(19.8)$ & & Ref & \\
\hline$>100$ farmers & 88 & $46(52.3)$ & & 4.43 & $2.68-7.34$ \\
\hline \multicolumn{3}{|c|}{ Number of workers directly involved in processing operation } & $p=0.001$ & & \\
\hline$\leq 150$ workers & 220 & $75(34.1)$ & & Ref & \\
\hline$>150$ workers & 176 & $32(18.2)$ & & 0.43 & $0.27-0.70$ \\
\hline \multicolumn{3}{|c|}{ Average waiting period from arrival at plant to processing } & $p=0.95$ & & \\
\hline$\leq 10 \mathrm{~h}$ & 308 & $83(26.9)$ & & Ref & \\
\hline$>10 \mathrm{~h}$ & 88 & $24(27.3)$ & & 1.01 & $0.60-1.73$ \\
\hline \multicolumn{3}{|c|}{ Average mortality rate $(\%)$ of birds on arrival at plant } & $p=0.001$ & & \\
\hline$<0.50$ & 176 & $32(18.2)$ & & Ref & \\
\hline$\geq 0.50$ & 220 & $75(34.1)$ & & 2.32 & $1.45-3.74$ \\
\hline \multicolumn{3}{|c|}{ Handling of sick/diseased birds } & $p<0.001$ & & \\
\hline Rejected at farm & 308 & $61(19.8)$ & & Ref & \\
\hline Processed last & 88 & $46(52.3)$ & & 4.43 & $2.68-7.34$ \\
\hline \multicolumn{3}{|l|}{ Use of pre-chiller } & $p=0.001$ & & \\
\hline Yes & 220 & $75(34.1)$ & & Ref & \\
\hline No & 176 & $32(18.2)$ & & 2.32 & $1.45-3.74$ \\
\hline \multicolumn{3}{|c|}{ Agents used in pre-chiller ${ }^{b}$} & $p=0.11$ & & \\
\hline Citric acid + chlorine & 88 & $24(27.3)$ & & Ref & \\
\hline No agents added & 132 & $51(38.6)$ & & 1.67 & $0.94-3.02$ \\
\hline \multicolumn{3}{|c|}{ Temperature of pre-chiller ${ }^{b}$} & $p<0.001$ & & \\
\hline Room temperature & 88 & $46(52.3)$ & & Ref & \\
\hline $10^{\circ} \mathrm{C}$ & 44 & $5(11.4)$ & & 0.11 & $0.04-0.33$ \\
\hline $20^{\circ} \mathrm{C}$ & 88 & $24(27.3)$ & & 0.34 & $0.18-0.64$ \\
\hline \multicolumn{3}{|l|}{ Agents used in chiller } & $p=0.01$ & & \\
\hline Chlorine & 352 & $102(29.0)$ & & 3.18 & $1.22-8.30$ \\
\hline No agents added ${ }^{c}$ & 44 & $5(11.4)$ & & Ref & \\
\hline \multicolumn{3}{|c|}{ Concentration of chlorine used in chiller ${ }^{c}$} & $p=0.79$ & & \\
\hline 20 ppm & 88 & $24(27.3)$ & & Ref & \\
\hline $21-50 \mathrm{ppm}$ & 264 & $78(29.5)$ & & 1.11 & $0.65-1.92$ \\
\hline \multicolumn{3}{|l|}{ Temperature of chiller } & $p=0.14$ & & \\
\hline$<1^{\circ} \mathrm{C}$ & 132 & $29(22.0)$ & & Ref & \\
\hline $1-4{ }^{\circ} \mathrm{C}$ & 264 & $78(29.5)$ & & 1.49 & $0.91-2.43$ \\
\hline \multicolumn{3}{|c|}{ Agents used for general cleaning of plant during processing } & $p=0.01$ & & \\
\hline Sanitizer & 352 & $102(29.0)$ & & Ref & \\
\hline Hot water only & 44 & $5(11.4)$ & & 0.31 & $0.12-0.82$ \\
\hline Worker segregation ${ }^{d}$ & & & $p=0.01$ & & \\
\hline Yes & 352 & $102(29.0)$ & & 3.18 & $1.22-8.30$ \\
\hline No & 44 & $5(11.4)$ & & Ref & \\
\hline
\end{tabular}

a Based on weekly throughput, small $<100,000$ birds; medium 101,000-160,000 birds; large $>161,000$ birds. ${ }^{b}$ Only 3 plants use pre-chillers. ${ }^{c}$ Only 3 plants add additional chlorine to chiller water. Chlorine concentration ranged from 1-5 ppm in the municipal water supply.

${ }^{\mathrm{d}}$ Colour coding of workers was done to limit movement of workers to prevent cross contamination of dirty and clean work areas.

\subsection{Multivariate Logistic Regression of Risk Factors for Isolation of Salmonella}

Of the nine variables included in the initial logistic regression model, only the average number of contract farmers, the number of workers directly involved in the processing, and the waiting period were retained in the final model. Processing plants with more than 100 contract farms were significantly associated with increased odds of Salmonella isolation (OR $\left.=8.5 ; \chi^{2}=16.968, p<0.001\right)$ (Table 3). Similarly, plants where the waiting period between arrival and slaughter was more than $10 \mathrm{~h}$ were significantly associated with Salmonella isolation $(\mathrm{OR}=2.9 ; \times 2=4.072, p=0.044)$. Plants, where there were more 
than 150 workers directly involved in processing, were included in the model but were not a significant predictor in the equation $(p=0.284)$. The Hosmer-Lemeshow test of goodness-of-fit test was not significant $\left(\chi^{2}=0.00, p=1\right)$, showing that the final logistic regression model fitted the data well.

Table 3. Results of a multivariate logistic regression of risk factors for Salmonella isolation from carcasses sampled at broiler processing plants in Trinidad.

\begin{tabular}{|c|c|c|c|c|c|c|c|}
\hline \multirow{2}{*}{ Variable } & \multirow{2}{*}{ Coef. } & \multirow{2}{*}{ Standard Error ${ }^{a}$} & \multirow{2}{*}{ Chi-Square } & \multirow{2}{*}{$p$-Value } & \multirow{2}{*}{ Odds Ratio } & \multicolumn{2}{|c|}{$95.0 \% \mathrm{CI}$} \\
\hline & & & & & & Lower & Upper \\
\hline$>100$ versus $\leq 100$ farmers & 2.145 & 0.521 & 16.968 & $<0.001$ & 8.543 & 3.078 & 23.707 \\
\hline$>150$ versus $\leq 150$ workers & 0.55 & 0.514 & 1.147 & 0.284 & 1.733 & 0.633 & 4.744 \\
\hline $\begin{array}{c}>10 \mathrm{~h} \text { versus } \leq 10 \mathrm{~h} \\
\text { waiting period }\end{array}$ & 1.073 & 0.532 & 4.072 & 0.044 & 2.925 & 1.031 & 8.296 \\
\hline Constant & -2.054 & 0.475 & 18.7 & $<0.001$ & 0.128 & & \\
\hline
\end{tabular}

\subsection{Isolation from Different Broiler Processing Plants and Types of Samples}

Overall, the isolation rate of Salmonella in carcasses sampled at broiler processing plants was $27.0 \%(107 / 396)$ (Table 4). Among all the samples collected during broiler processing, the isolation rate of Salmonella was highest in pre-evisceration carcasses (51.1\%; $23 / 45)$ followed by chilled whole carcasses $(44.4 \%$; $20 / 45)$, chilled chicken parts $(40.0 \%$; $18 / 45)$, and post-evisceration carcasses $(37.8 \% ; 17 / 45)$ (Table 4$)$. Salmonella was detected only in $2.2 \%(2 / 90)$ and $5.6 \%(2 / 36)$ cloacal swabs and immersion chiller water samples, respectively. 
Table 4. Frequency of isolation of Salmonella by type of samples tested at each plant.

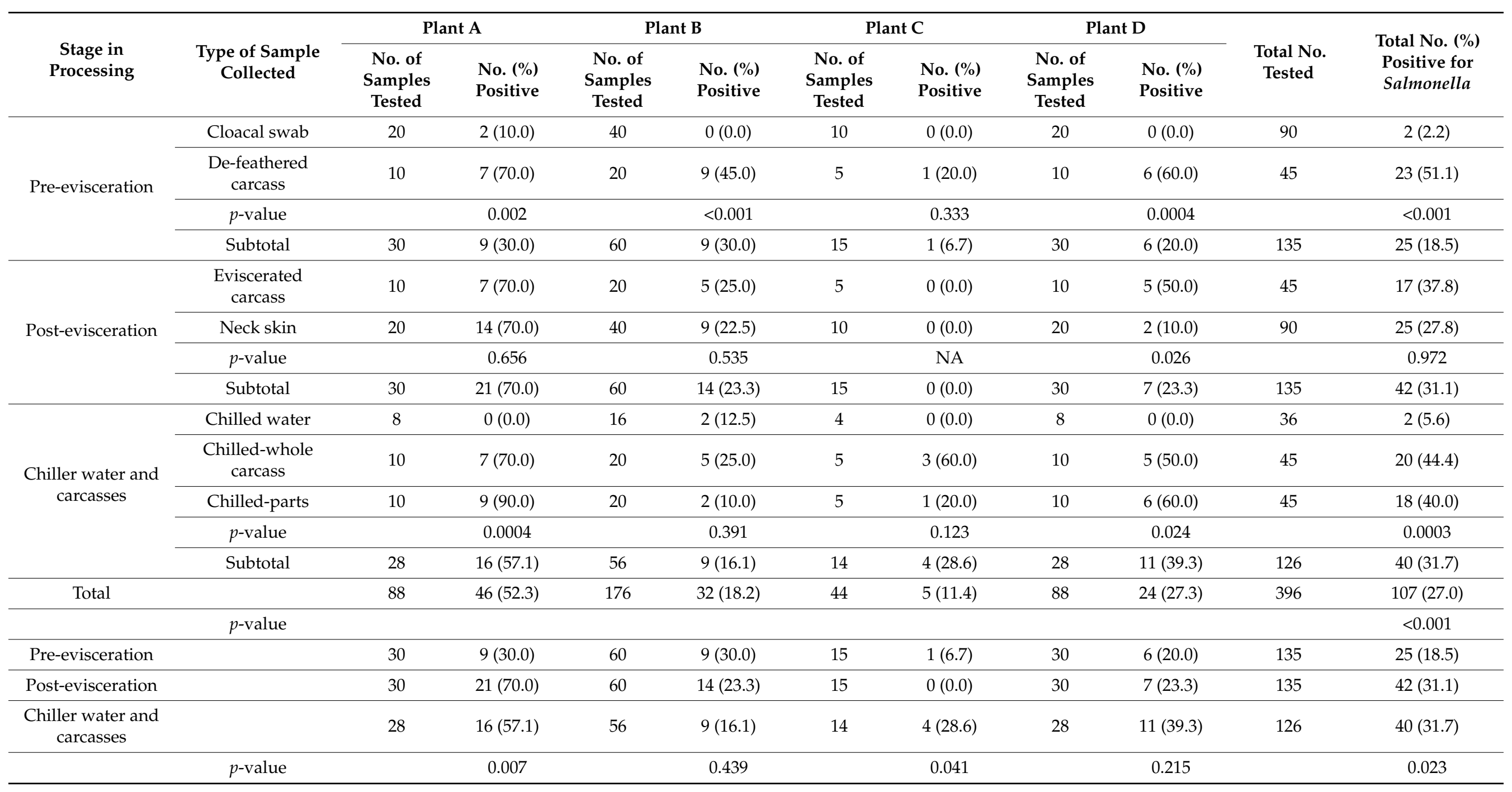




\subsection{Serotypes of Salmonella Isolates}

S. enterica serotype Enteritidis $(20.8 \% ; 15 / 72)$, Javiana $(16.7 \% ; 12 / 72)$, and Infantis $(12.5 \% ; 9 / 72)$ were the most prevalent among a total of 16 different serotypes isolated at broiler processing plants (Table 5). Serotypes Kentucky, Anatum, Schwarzengrund, and Albany were found in less than $10 \%$ of the isolates. Only one isolate each of serotypes Hindmarsh, Madjorio, Mbandaka, S. enterica subspecies Houtenae, Virchow, Weltevrden, Aberdeen, Alachua, and Ayinde were detected among all the isolates. Serotype Enteritidis was found primarily (14/15 samples: $93.3 \%)$ in chilled whole and chicken parts as well as neck skins.

Table 5. Salmonella serotypes isolated from different types of samples.

\begin{tabular}{|c|c|c|c|}
\hline Stage of Processing & $\begin{array}{l}\text { No. of Samples } \\
\text { Positive for } \\
\text { Salmonella }\end{array}$ & $\begin{array}{l}\text { No. }(\%)^{a} \text { of } \\
\text { Isolates } \\
\text { Serotyped }\end{array}$ & Serotypes (No., \%) \\
\hline Cloacal swabs & 2 & $0(0.0)$ & Not applicable \\
\hline Pre-evisceration carcass & 23 & $2(8.7)$ & $\begin{array}{l}\text { Weltevreden }(1,50.0) \\
\text { Enteritidis }(1,50.0)\end{array}$ \\
\hline Post-evisceration carcass & 17 & $5(29.4)$ & $\begin{array}{l}\text { Javiana }(3,60.0) \\
\text { Virchow }(1,20.0) \\
\text { Infantis }(1,20.0)\end{array}$ \\
\hline Neck skins & 25 & $25(100.0)$ & $\begin{array}{c}\text { Javiana }(7,28.0) \\
\text { Schwarzengrund }(5,20.0) \\
\text { Albany }(4,16.0) \\
\text { Anatum }(3,12.0) \\
\text { Infantis }(2,8.0) \\
\text { Group C2 }{ }^{\mathrm{b}}(2,8.0) \\
\text { Madjorio }(1,4.0) \\
\text { Enteritidis }(1,4.0)\end{array}$ \\
\hline Chiller water & 2 & $2(100.0)$ & $\begin{array}{c}\text { Salmonella spp. }(1,50.0) \\
\text { subspecies Houtenae IV }(1,50.0)\end{array}$ \\
\hline Chilled whole carcass & 20 & $20(100.0)$ & $\begin{array}{c}\text { Enteritidis }(7,35.0) \\
\text { Infantis }(4,20.0) \\
\text { Anatum }(2,10.0) \\
\text { Albany }(1,5.0) \\
\text { Mbandaka }(1,5.0) \\
\text { Schwarzengrund }(1,5.0) \\
\text { Aberdeen }(1,5.0) \\
\text { Javiana }(1,5.0) \\
\text { Kentucky }(1,5.0) \\
\text { Ayinde }(1,5.0)\end{array}$ \\
\hline Chilled chicken parts & 18 & $18(100.0)$ & $\begin{array}{c}\text { Enteritidis }(6,33.3) \\
\text { Kentucky }(6,33.3) \\
\text { Infantis }(2,11.1) \\
\text { Hindmarsh }(1,5.6) \\
\text { Javiana }(1,5.6) \\
\text { Anatum }(1,5.6) \\
\text { Alachua }(1,5.6)\end{array}$ \\
\hline Total & 107 & $72(67.3)$ & \\
\hline
\end{tabular}

a Of the number of randomly selected Salmonella serotypes from each source; ${ }^{\mathrm{b}}$ Serogroup (Group C2) could not be determined to the serotype level.

\subsection{Frequency of Resistance of Salmonella Isolates to Eight Antimicrobial Agents at Different Processing Plants}

The prevalence of resistance to antimicrobial agents among Salmonella isolates tested was $90.5 \%(114 / 126)$ as resistance was exhibited to one or more of the eight antimicrobial agents tested (Figure 2). Overall, resistance was relatively high to K (85.7\%) and DO (74.6\%) but relatively low to SXT $(0.8 \%), \mathrm{C}(0.8 \%)$, and AMC $(2.4 \%)$. The differences were statistically significant $(p<0.05)$. The overall prevalence of resistance to antimicrobial agents by 
Salmonella isolates was 96.7\% (58/61), 97.1\% (33/34), 50.0\% (3/6), and 80.0\% (20/25) at plant A, B, C, and D, respectively, and these differences were statistically significant $(p<0.05)$.

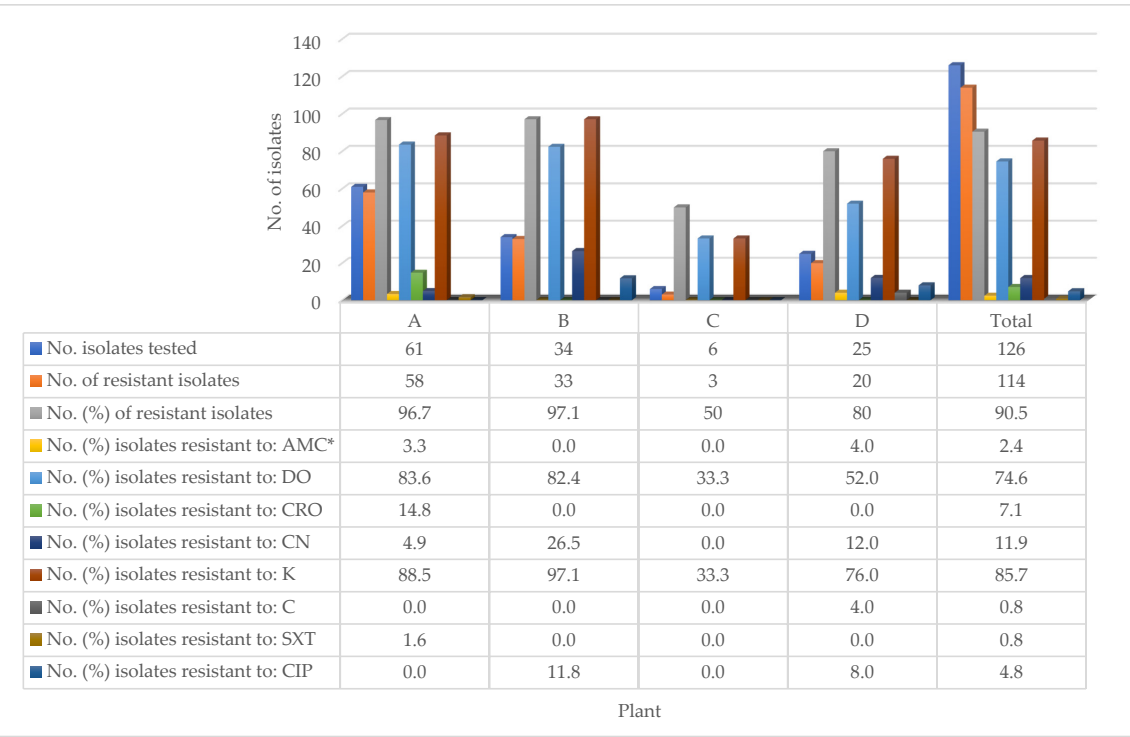

Figure 2. Antimicrobial resistance of Salmonella isolates isolated from four processing plants. * AMC, amoxicillin-clavulanic acid (30 $\mu \mathrm{g}) ; \mathrm{DO}$, doxycycline $(30 \mu \mathrm{g}) ; \mathrm{CRO}$, ceftriaxone (30 $\mu \mathrm{g})$; CN, gentamicin $(10 \mu \mathrm{g}) ; \mathrm{K}$, kanamycin $(30 \mu \mathrm{g})$; C, chloramphenicol $(30 \mu \mathrm{g})$; SXT, sul-famethoxazole-trimethoprim (23.75 and $1.25 \mu \mathrm{g}) ; \mathrm{CIP}$, ciprofloxacin $(5 \mu \mathrm{g})$.

\subsection{Frequency of Antimicrobial Resistance of Salmonella Isolates Based on the Type of Sample}

The frequencies of resistance to antimicrobial agents (Table 6) were similar amongst the various types of samples, ranging from $86.2 \%$ to $100 \%$ in chilled chicken parts, postevisceration carcasses, chilled whole carcasses, neck skins, pre-evisceration carcasses, chiller water, and cloacal swabs. The frequency of resistance to DO was significantly $(p=0.045)$ higher for isolates of Salmonella that originated from pre-evisceration carcasses $(23 / 25$, $92.0 \%$ ) compared with isolates from other types of samples. The differences in the frequency of resistance were not statistically significant $(p>0.05)$ for Salmonella isolated from the other types of samples other than from chiller water samples.

Table 6. Antimicrobial resistance of Salmonella isolated from various stages of processing.

\begin{tabular}{|c|c|c|c|c|c|c|c|c|c|c|c|}
\hline \multirow{2}{*}{$\begin{array}{l}\text { Stage in } \\
\text { Processing }\end{array}$} & \multirow{2}{*}{$\begin{array}{l}\text { Type of } \\
\text { Sample } \\
\text { Collected }\end{array}$} & \multirow{2}{*}{$\begin{array}{l}\text { No. of } \\
\text { Isolates } \\
\text { Tested }\end{array}$} & \multirow{2}{*}{$\begin{array}{c}\text { No. }(\%) \text { of } \\
\text { Isolates } \\
\text { Resistant }\end{array}$} & \multicolumn{8}{|c|}{ No. (\%) Resistant to ${ }^{b}$ : } \\
\hline & & & & AMC & DO & CRO & $\mathrm{CN}$ & $\mathbf{K}$ & $C$ & SXT & CIP \\
\hline \multirow{4}{*}{ Pre-evisceration } & Cloacal swab & 3 & $3(100.0)$ & $\begin{array}{c}0 \\
(0.0)\end{array}$ & $\begin{array}{c}1 \\
(33.3)\end{array}$ & $\begin{array}{c}0 \\
(0.0)\end{array}$ & $\begin{array}{c}1 \\
(33.3)\end{array}$ & $\begin{array}{c}3 \\
(100.0)\end{array}$ & $\begin{array}{c}0 \\
(0.0)\end{array}$ & $\begin{array}{c}0 \\
(0.0)\end{array}$ & $\begin{array}{c}0 \\
(0.0)\end{array}$ \\
\hline & $\begin{array}{l}\text { Defeathered } \\
\text { carcass }\end{array}$ & 25 & $24(96.0)$ & $\begin{array}{c}0 \\
(0.0)\end{array}$ & $\begin{array}{c}23 \\
(92.0)\end{array}$ & $\begin{array}{c}1 \\
(4.0)\end{array}$ & $\begin{array}{c}2 \\
(8.0)\end{array}$ & $\begin{array}{c}23 \\
(92.0)\end{array}$ & $\begin{array}{c}0 \\
(0.0)\end{array}$ & $\begin{array}{c}0 \\
(0.0)\end{array}$ & $\begin{array}{c}0 \\
(0.0)\end{array}$ \\
\hline & $p$-value & & 1 & NA & 0.045 & 1 & 0.298 & 1 & NA & NA & NA \\
\hline & Subtotal & 28 & $27(96.4)$ & $\begin{array}{c}0 \\
(0.0)\end{array}$ & $\begin{array}{c}24 \\
(85.7)\end{array}$ & $\begin{array}{c}1 \\
(3.6)\end{array}$ & $\begin{array}{c}3 \\
(10.7)\end{array}$ & $\begin{array}{c}26 \\
(92.9)\end{array}$ & $\begin{array}{c}0 \\
(0.0)\end{array}$ & $\begin{array}{c}0 \\
(0.0)\end{array}$ & $\begin{array}{c}0 \\
(0.0)\end{array}$ \\
\hline \multirow{4}{*}{ Post-evisceration } & $\begin{array}{c}\text { Eviscerated } \\
\text { Carcass }\end{array}$ & 17 & $15(88.2)$ & $\begin{array}{c}0 \\
(0.0)\end{array}$ & $\begin{array}{c}12 \\
(70.6)\end{array}$ & $\begin{array}{c}0 \\
(0.0)\end{array}$ & $\begin{array}{c}2 \\
(11.8)\end{array}$ & $\begin{array}{c}14 \\
(82.4)\end{array}$ & $\begin{array}{c}0 \\
(0.0)\end{array}$ & $\begin{array}{c}0 \\
(0.0)\end{array}$ & $\begin{array}{c}0 \\
(0.0)\end{array}$ \\
\hline & Neck skin & 25 & $23(92.0)$ & $\begin{array}{c}0 \\
(0.0)\end{array}$ & $\begin{array}{c}18 \\
(72.0)\end{array}$ & $\begin{array}{c}2 \\
(8.0)\end{array}$ & $\begin{array}{c}6 \\
(24.0)\end{array}$ & $\begin{array}{c}21 \\
(84.0)\end{array}$ & $\begin{array}{c}0 \\
(0.0)\end{array}$ & $\begin{array}{c}0 \\
(0.0)\end{array}$ & $\begin{array}{c}3 \\
(12.0)\end{array}$ \\
\hline & $p$-value & & 1 & NA & 1 & 0.506 & 0.439 & 1 & NA & NA & 0.260 \\
\hline & Subtotal & 42 & $38(90.5)$ & $\begin{array}{c}0 \\
(0.0)\end{array}$ & $\begin{array}{c}30 \\
(71.4)\end{array}$ & $\begin{array}{c}2 \\
(4.8)\end{array}$ & $\begin{array}{c}8 \\
(19.0)\end{array}$ & $\begin{array}{c}35 \\
(83.3)\end{array}$ & $\begin{array}{c}0 \\
(0.0)\end{array}$ & $\begin{array}{c}0 \\
(0.0)\end{array}$ & $\begin{array}{c}3 \\
(7.1)\end{array}$ \\
\hline
\end{tabular}


Table 6. Cont.

\begin{tabular}{|c|c|c|c|c|c|c|c|c|c|c|c|}
\hline \multirow{2}{*}{$\begin{array}{c}\text { Stage in } \\
\text { Processing }\end{array}$} & \multirow{2}{*}{$\begin{array}{c}\text { Type of } \\
\text { Sample } \\
\text { Collected }\end{array}$} & \multirow{2}{*}{$\begin{array}{c}\text { No. of } \\
\text { Isolates } \\
\text { Tested } \\
\end{array}$} & \multirow{2}{*}{$\begin{array}{c}\text { No. }(\%) \text { of } \\
\text { Isolates } \\
\text { Resistant }^{\text {a }}\end{array}$} & \multicolumn{8}{|c|}{ No. $(\%)$ Resistant to ${ }^{b}$ : } \\
\hline & & & & AMC & DO & CRO & $\mathrm{CN}$ & $\mathbf{K}$ & $\mathrm{C}$ & SXT & CIP \\
\hline \multirow{5}{*}{$\begin{array}{l}\text { Chiller water and } \\
\text { carcasses }\end{array}$} & Chiller water & 2 & $2(100.0)$ & $\begin{array}{c}0 \\
(0.0) \\
\end{array}$ & $0(0.0)$ & $\begin{array}{c}0 \\
(0.0) \\
\end{array}$ & $\begin{array}{c}0 \\
(0.0) \\
\end{array}$ & $\begin{array}{c}2 \\
(100.0) \\
\end{array}$ & $\begin{array}{c}0 \\
(0.0) \\
\end{array}$ & $\begin{array}{c}0 \\
(0.0) \\
\end{array}$ & $\begin{array}{c}1 \\
(50.0) \\
\end{array}$ \\
\hline & $\begin{array}{l}\text { Chilled-whole } \\
\text { carcass }\end{array}$ & 29 & $25(86.2)$ & $\begin{array}{c}0 \\
(0.0) \\
\end{array}$ & $\begin{array}{c}20 \\
(69.0)\end{array}$ & $\begin{array}{c}4 \\
(13.8)\end{array}$ & $\begin{array}{c}4 \\
(13.8)\end{array}$ & $\begin{array}{c}24 \\
(82.8)\end{array}$ & $\begin{array}{c}0 \\
(0.0) \\
\end{array}$ & $\begin{array}{c}0 \\
(0.0)\end{array}$ & $\begin{array}{c}2 \\
(6.9)\end{array}$ \\
\hline & Chilled-parts & 25 & $22(88.0)$ & $\begin{array}{c}3 \\
(12.0)\end{array}$ & $\begin{array}{c}20 \\
(80.0)\end{array}$ & $\begin{array}{c}2 \\
(8.0)\end{array}$ & $\begin{array}{c}0 \\
(0.0) \\
\end{array}$ & $\begin{array}{c}21 \\
(84.0)\end{array}$ & $\begin{array}{c}1 \\
(4.0) \\
\end{array}$ & $\begin{array}{c}1 \\
(4.0)\end{array}$ & $\begin{array}{c}0 \\
(0.0)\end{array}$ \\
\hline & $p$-value & & 0.846 & 0.140 & 0.050 & 0.698 & 0.135 & 0.814 & 0.532 & 0.532 & 0.009 \\
\hline & Subtotal & 56 & 49 (87.5) & $\begin{array}{c}3 \\
(5.4) \\
\end{array}$ & $\begin{array}{c}40 \\
(71.4)\end{array}$ & $\begin{array}{c}6 \\
(10.7) \\
\end{array}$ & $\begin{array}{c}4 \\
(7.1) \\
\end{array}$ & $\begin{array}{c}47 \\
(83.9) \\
\end{array}$ & $\begin{array}{c}1 \\
(1.8) \\
\end{array}$ & $\begin{array}{c}1 \\
(1.8) \\
\end{array}$ & $\begin{array}{c}3 \\
(5.4) \\
\end{array}$ \\
\hline Total & & $126^{\mathrm{c}}$ & $114(90.5)$ & $\begin{array}{c}3 \\
(2.4) \\
\end{array}$ & $\begin{array}{c}94 \\
(74.6)\end{array}$ & $\begin{array}{c}9 \\
(7.1) \\
\end{array}$ & $\begin{array}{c}15 \\
(11.9) \\
\end{array}$ & $\begin{array}{c}108 \\
(85.7)\end{array}$ & $\begin{array}{c}1 \\
(0.8) \\
\end{array}$ & $\begin{array}{c}1 \\
(0.8) \\
\end{array}$ & $\begin{array}{c}6 \\
(4.8) \\
\end{array}$ \\
\hline Pre-evisceration & & 28 & $27(96.4)$ & $\begin{array}{c}0 \\
(0.0)\end{array}$ & $\begin{array}{c}24 \\
(85.7)\end{array}$ & $\begin{array}{c}1 \\
(3.6)\end{array}$ & $\begin{array}{c}3 \\
(10.7)\end{array}$ & $\begin{array}{c}26 \\
(92.9) \\
\end{array}$ & $\begin{array}{c}0 \\
(0.0) \\
\end{array}$ & $\begin{array}{c}0 \\
(0.0)\end{array}$ & $\begin{array}{c}0 \\
(0.0)\end{array}$ \\
\hline Post-evisceration & & 42 & 38 (90.5) & $\begin{array}{c}0 \\
(0.0) \\
\end{array}$ & $\begin{array}{c}30 \\
(71.4) \\
\end{array}$ & $\begin{array}{c}2 \\
(4.8) \\
\end{array}$ & $\begin{array}{c}8 \\
(19.0) \\
\end{array}$ & $\begin{array}{c}35 \\
(83.3) \\
\end{array}$ & $\begin{array}{c}0 \\
(0.0) \\
\end{array}$ & $\begin{array}{c}0 \\
(0.0) \\
\end{array}$ & $\begin{array}{c}3 \\
(7.1) \\
\end{array}$ \\
\hline $\begin{array}{c}\text { Chiller water and } \\
\text { carcasses }\end{array}$ & & 56 & 49 (87.5) & $\begin{array}{c}3 \\
(5.4)\end{array}$ & $\begin{array}{c}40 \\
(71.4)\end{array}$ & $\begin{array}{c}6 \\
(10.7)\end{array}$ & $\begin{array}{c}4 \\
(7.1) \\
\end{array}$ & $\begin{array}{c}47 \\
(83.9)\end{array}$ & $\begin{array}{c}1 \\
(1.8) \\
\end{array}$ & $\begin{array}{c}1 \\
(1.8)\end{array}$ & $\begin{array}{c}3 \\
(5.4)\end{array}$ \\
\hline & $p$-value & & 0.422 & 0.147 & 0.310 & 0.373 & 0.193 & 0.471 & 0.533 & 0.533 & 0.374 \\
\hline
\end{tabular}

${ }^{a}$ Resistance to one or more agents tested. ${ }^{b}$ AMC, amoxicillin-clavulanic acid $(30 \mu \mathrm{g}) ; \mathrm{DO}$, doxycycline $(30 \mu \mathrm{g}) ; \mathrm{CRO}$, ceftriaxone $(30 \mu \mathrm{g})$; $\mathrm{CN}$, gentamicin $(10 \mu \mathrm{g}) ; \mathrm{K}$, kanamycin $(30 \mu \mathrm{g})$; C, chloramphenicol $(30 \mu \mathrm{g})$; SXT, sulfamethoxazole-trimethoprim $(23.75 \mathrm{and} 1.25 \mu \mathrm{g})$; CIP, ciprofloxacin $(5 \mu \mathrm{g})$. ${ }^{\mathrm{c}}$ A total of 126 isolates may have included duplicates of isolates obtained from TT/XLT-4, TT/BGA, RVS/XLT-4, and RVS/BGA media, solely of phenotypes. NA: Not applicable.

\subsection{Resistance of Salmonella Isolates Based on Serotype}

Sixteen different serotypes of Salmonella were identified from the 72 isolates subjected to conventional serotyping. Serotypes Enteritidis and Javiana were the most prevalent serotypes with $60.0 \%(9 / 15)$ and $83.3 \%(10 / 12)$ exhibiting resistance to one or more agents, respectively (Table 7$)$. All isolates (100.0\%) belonging to serotypes Albany, Anatum, and Kentucky; $88.9 \%$ for Infantis, $83.3 \%$ for Javiana, and $83.3 \%$ for Schwarzengrund exhibited resistance to antimicrobial agents. Amongst the different serotypes, the differences in the resistance exhibited were only statistically significant to DO $(p<0.001)$.

Table 7. Resistance exhibited by different serotypes isolated at four processing plants.

\begin{tabular}{|c|c|c|c|c|c|c|c|c|c|c|}
\hline \multirow{2}{*}{ Serotype $^{a}$} & \multirow{2}{*}{$\begin{array}{c}\text { No. of } \\
\text { Isolates } \\
\text { Tested } \\
\end{array}$} & \multirow{2}{*}{$\begin{array}{c}\text { No. }(\%) \text { of } \\
\text { Isolates } \\
\text { Resistant }\end{array}$} & \multicolumn{8}{|c|}{ No. (\%) Isolates Resistant to ${ }^{c}$ : } \\
\hline & & & AMC & DO & CRO & $\mathrm{CN}$ & $\mathbf{K}$ & C & SXT & CIP \\
\hline Albany & 5 & $5(100.0)$ & $0(0.0)$ & $5(100.0)$ & $2(40.0)$ & $0(0.0)$ & $4(80.0)$ & $0(0.0)$ & $0(0.0)$ & $0(0.0)$ \\
\hline Anatum & 6 & $6(100.0)$ & $0(0.0)$ & $6(100.0)$ & $2(33.3)$ & $0(0.0)$ & $4(66.7)$ & $0(0.0)$ & $0(0.0)$ & $0(0.0)$ \\
\hline Enteritidis & 15 & $9(60.0)$ & $1(6.7)$ & $1(6.7)$ & $0(0.0)$ & $0(0.0)$ & $9(60.0)$ & $1(6.7)$ & $0(0.0)$ & $2(13.3)$ \\
\hline Infantis & 9 & $8(88.9)$ & $0(0.0)$ & $6(66.7)$ & $0(0.0)$ & $2(22.2)$ & $8(88.9)$ & $0(0.0)$ & $0(0.0)$ & $0(0.0)$ \\
\hline Javiana & 12 & $10(83.3)$ & $0(0.0)$ & $10(83.3)$ & $0(0.0)$ & $4(33.3)$ & $10(83.3)$ & $0(0.0)$ & $0(0.0)$ & $3(25.0)$ \\
\hline Kentucky & 7 & $7(100.0)$ & $2(28.6)$ & $7(100.0)$ & $1(14.3)$ & $0(0.0)$ & $6(85.7)$ & $0(0.0)$ & $1(14.3)$ & $0(0.0)$ \\
\hline Schwarzengrund & 6 & $5(83.3)$ & $0(0.0)$ & $4(66.7)$ & $0(0.0)$ & $2(33.3)$ & $5(83.3)$ & $0(0.0)$ & $0(0.0)$ & $0(0.0)$ \\
\hline$p$-value & & & 0.813 & $<0.001$ & 0.252 & 0.238 & 0.745 & 1 & 0.996 & 0.192 \\
\hline Total & 60 & $50(83.3)$ & $3(5.0)$ & $39(65.0)$ & $5(8.3)$ & 8 (13.3) & $46(76.7)$ & $1(1.7)$ & 1 (1.7) & $5(8.3)$ \\
\hline
\end{tabular}

${ }^{a}$ In addition, 2 (100.0\%) of 2 Group C2 isolates exhibited resistance to one or more of the eight antimicrobial agents tested; 1 (100.0\%) of 1 of the following serotypes Aberdeen, Alachua, Ayinde, Hindmarsh, Madjorio, Mbandaka, Salmonella sp. (untypable), S. Houtenae, Virchow, and Weltevreden were resistant, i.e., a total of 12 isolates. ${ }^{\mathrm{b}}$ Exhibited resistance to one or more antimicrobial agents. ${ }^{\mathrm{c}}$ AMC,

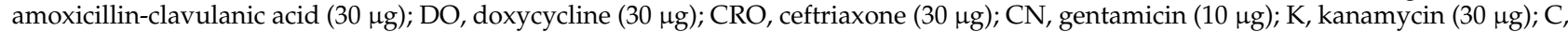
chloramphenicol (30 $\mu \mathrm{g})$; SXT, sulfamethoxazole-trimethoprim (23.75 and $1.25 \mu \mathrm{g})$; CIP, ciprofloxacin (5 $\mu \mathrm{g})$. 


\subsection{Antimicrobial Resistance Patterns}

A total of $14(12.3 \%)$ of the 114 isolates of Salmonella exhibited multidrug resistance, i.e., resistance to antimicrobial agents belonging to three or more classes. Overall, a total of 12 different patterns were observed consisting of DO-K, which was the predominant pattern, with $54.4 \%$ isolates exhibiting the resistance pattern. Resistance to $\mathrm{K}$ alone was exhibited by $15(13.2 \%)$ isolates, $12(10.5 \%)$ isolates exhibited resistance to DO-CN-K, 8 (7.0\%) exhibited resistance to DO-CRO-K, and $6(5.3 \%)$ were resistant to $\mathrm{DO}$ alone. Other patterns observed ranged from $0.9 \%$ to $2.6 \%$ of resistant isolates.

\section{Discussion}

This is considered the first cross-sectional study conducted in the broiler processing plants in Trinidad and Tobago that documented the frequency of isolation of Salmonella along the processing lines. The study also characterized the isolates regarding their serotypes and antimicrobial resistance to currently used antimicrobial agents in the poultry industry. The food safety importance of the study cannot be underestimated because the four processing plants operational in the country supply the majority of local chickens and chicken products sold at supermarkets.

Of food safety concern, is the high level of contamination found in pre-packaged chilled whole carcasses (44.4\%) and chilled chicken parts (40.0\%) across the four processing plants. Salmonellosis has been reported in humans who consume inadequately cooked Salmonella-contaminated chicken meat $[52,53]$. Our findings agree with the prevalence of Salmonella found in chilled chicken carcasses in abattoirs elsewhere, where 48.0\% [43], $45.2 \%$ [54], and 50.0\% [55] were reported in the United States, China, and Brazil, respectively. These findings were higher than the 8.3\% [56] and 3.75\% [57] reported in Iran and the Czech Republic, respectively. It is interesting to note that the most recent study on the prevalence of Salmonella in chickens that originated from commercial processing plants in Trinidad was $8.3 \%$ [38]. The differences in the prevalence have been reported to be affected by the carriage of Salmonella during de-feathering [57], evisceration, and spray washing steps [58] as well as by contaminated chiller water [59].

The strategy used in our study which included the collection of samples from the time of reception of live chickens to the finished chilled chickens longitudinally, from the preevisceration samples to chilled carcasses during each visit provided evidence of statistically significant $(p=0.023)$ increased levels of contamination along the stages of processing. The differences in the frequencies of isolation of Salmonella in the samples between and within the four processing plants, could be due in part, to the different management, production, and risk factors at these plants. These findings were not surprising because other studies have reported progressive increases in the frequency of contamination with Salmonella during processing $[60,61]$.

It is significant that the frequency of isolation of Salmonella from the cloacal swabs preslaughter across the four plants was $2.2 \%$ ranging from $0.0 \%$ to $10.0 \%$. This is an indication that the prevalence of Salmonella was relatively low on the poultry farms from where the slaughtered birds originated. Our findings agree with the prevalence of Salmonella in cloacal swabs of broilers pre-slaughter reported in Trinidad and Tobago, 3.95\% (3/76) [62]; Brazil, 7.0\% (7/100) [20]; and Colombia, 12.5\% (8/64) [63].

It was of epidemiological relevance to have detected that $71.4 \%$ of the 14 risk factors investigated demonstrated statistically significant association with the contamination of chicken carcasses during processing at the plants. Significantly higher frequencies of isolation of Salmonella were detected among the following factors including medium-sized plants, use of more than 100 contract farmers, employment of less than 150 workers directly involved in processing, the average mortality rate of over $0.5 \%$ in broilers on arrival at the plant, i.e., dead on arrival, the use of pre-chillers, and the use of sanitizers in chiller water, used sanitizers for general cleaning of plants, among other factors. Many of these risk factors have been documented to be associated with the isolation of Salmonella in processing plants by others $[15,64-67]$. Standardized sanitation protocols with surveillance 
to monitor the efficacy and the development of resistance is suggested. In addition, frequent training programs for processing plant workers and farmers to educate them on the current best-practices will be beneficial in reducing cross-contamination along the continuum. Interestingly, further regression analyses and the odds ratio (OR) revealed that Salmonella was 4.4 more likely (95\% CI: 2.68-7.34) to be isolated from chickens in plants that received birds from more than 100 farmers. This risk could be attributed to the increased possibility of slaughtering broilers from Salmonella-infected farms. Similarly, it was detected that plants that allowed the slaughter of chickens from batches with mortality rates of over $0.5 \%$ on arrival at the plants were 2.3 times more likely (95\% CI: $1.45-3.74)$ to lead to the isolation of Salmonella from chickens at those plants. Although the specific pathogens responsible for deaths experienced during transportation to the plant were not known, the possibility exists that Salmonella may be involved. The contamination of feathers of chickens from direct contact with feces of infected broilers shedding Salmonella and exposure to the pathogen in the transport vehicle on its way to the plant has been documented $[68,69]$. Similarly, the risk of contamination of chickens increased considerably by 4.4 times $(95 \%$ CI: 2.68-7.34) in plants that permitted the slaughter of sick birds, albeit being processed last instead of being rejected at farms. The possibility of seeding the plant environment with pathogens, including Salmonella, is pertinent, if the cleaning of the plant is inadequate. Salmonella was isolated at a significantly higher frequency in plants that used chlorine $(29.0 \%)$ than those that used hot water $(11.4 \%)$. This is because Salmonella has been reported to develop resistance to sanitizers [70-72]. Additionally, our study noted that plants that used pre-chillers but did not add chemical agents were found to be 1.7 times more likely (95\% CI: 0.94-3.02) to result in the recovery of Salmonella. The proper use of chillers and sanitizers in processing plants can therefore not be ignored [73,74].

In our study, the WCE method yielded a statistically significant higher $(53.9 \%)$ frequency of isolation of Salmonella than either the WCR (35.0\%) or the NS (42.2.\%) methods, making it the most sensitive method for Salmonella detection as reported by others $[43,75]$. Berrang et al. [36] attributed this increased sensitivity to the ability of the WCE method to facilitate the proliferation of Salmonella in low quantities or those firmly attached to the skin of the chicken. However, the challenges associated with WCE method, particularly the considerably larger incubator space requirement compared with the use of WCR and NS methods, cannot be disregarded thereby making it an impractical method for routine surveillance testing but applicable as a research tool. It has been reported that the types of samples and the methods of enrichment affect their sensitivities to detect Salmonella in chickens [76,77].

The predominant serotypes of Salmonella isolated were Enteritidis, Javiana, and Infantis. These serotypes have similarly been isolated from chicken-associated samples in the country, such as chickens sampled from supermarkets that originated from broiler processing plants and outlets of cottage poultry processors [38] and chicken layers [78]. In the current study, it was found that the serotypes were detected at different frequencies from the types of samples tested in the processing plants, a finding that agrees with published reports $[79,80]$. Of food safety and public health, the significance is the fact that some of these predominant serotypes were determined in the Caribbean Public Health Agency (CARPHA) State of Public Health report [81], to be amongst the top 15 human Salmonella serotypes detected in the region. Similarly, the predominant serotypes in our study were also reported to be the most commonly Salmonella serotypes associated with human salmonellosis in Trinidad and Tobago between 2005-2012 [81]. It cannot be underestimated that serotype Enteritidis has globally been associated with poultry meat and eggs, and responsible for human cases and epidemics of salmonellosis [82,83].

The high prevalence of resistance $(90.5 \%)$ to antimicrobial agents by the 126 isolates of Salmonella recovered from the four processing plants, has both zoonotic and therapeutic implications. It is important to have detected that the high prevalence of resistance was exhibited to antimicrobial agents routinely used in the poultry industry in the country. It has been reported that zoonotic spread of Salmonella to workers at the commercial processing 
plants may occur $[84,85]$ and as well as therapeutic failure in consumers of improperly cooked chickens contaminated by antimicrobial resistant-Salmonella [80]. Similarly, a high prevalence of resistance to antimicrobial agents $(100.0 \%)$ has been reported in chilled chickens from supermarkets and cottage poultry processors [39]. Although the current study was not farm-based, the prevalence of resistant Salmonella in chickens processed at the plants may be indicative of the level of resistance of Salmonella on the contract farms from where they originated. It has been documented that the misuse or over-use of antimicrobial agents by farmers may result in the development of resistance to antimicrobial agents [86]. This is a common practice particularly in developing countries, including Trinidad and Tobago, where although laws governing the type and use of antimicrobial agents for prophylaxis, growth promotion, and therapy exist, prevailing challenges limit or prevent their enforcement $[87,88]$.

With regard to the eight antimicrobial agents tested, it was important that the overall prevalence of resistance was comparatively low (0.8-11.9\%) to six (amoxicillin-clavulanic acid, ceftriaxone, gentamicin, chloramphenicol, sulphamethoxazole-trimethoprim, and ciprofloxacin) of the antimicrobial agents, while significantly higher prevalence was exhibited to doxycycline $(74.6 \%)$ and kanamycin $(85.7 \%)$. Furthermore, the study found that the prevalence of resistance to the antimicrobial agents varied significantly across the processing plants from where the Salmonella isolates originated. These findings reflect the differences in the types and the frequency of use of antimicrobial agents on the contract farms that supplied live broilers to the plants. The high prevalence of resistance exhibited to doxycycline and kanamycin has been documented in chickens in the country [39]. The detection of a high prevalence of resistance $(60.0 \%$ to $88.9 \%)$ among the top three detected serotypes (Enteritidis, Javiana, and Infantis) may also be therapeutic significance to infected broilers or humans. Differences in the prevalence of resistance to antimicrobial agents by Salmonella have been reported to vary among serotypes of Salmonella from chickens by others $[71,89]$. Therefore, there is a need to monitor the use of the two antimicrobial agents on broiler farms in the country.

It is concluded that the high prevalence of Salmonella (27.0\%) including antimicrobialresistant strains $(90.5 \%)$, along with the predominance of three serotypes (Enteritidis, Javiana, and Infantis) among the isolates has implications for human salmonellosis in the country. The relative risk of salmonellosis posed by consumption of under-cooked Salmonella-contaminated chicken meat from these plants needs to be emphasized. The fact that 10 of the 14 risk factors investigated were statistically significantly associated with the contamination of chicken in the processing lines along with the odds ratio (OR) generated provides critical control points where interventions may be successfully applied. Our study reveals that the WCE method, which is not used for routine surveillance of Salmonella in chickens, demonstrated its significantly higher sensitivity when compared with either the WCR or NS methods, a finding that may be indicative of the potential under-reporting of the prevalence of antimicrobial resistant Salmonella in chickens in the country. The high prevalence of antimicrobial resistance exhibited by Salmonella isolates in this study poses both zoonotic and therapeutic implications to humans exposed to infected chickens. It is imperative to control the use of antimicrobial agents on poultry farms to reduce the development of antimicrobial resistance among Salmonella.

Supplementary Materials: Available online at https:/ /www.mdpi.com/article/10.3390/microorganisms9 051048/s1: S1: Flow chart of activities that take place at the four processing plants, S2: Broiler processing plant questionnaire.

Author Contributions: Conceptualization: A.A.A. and K.G.; methodology: A.S.K., A.A.A., W.A., S.R., and K.G.; validation: A.A.A., K.G., A.S.K., and W.A.; formal analysis: K.G., A.S.K., and A.A.A.; investigation: S.R., A.S.K., and A.A.A.; resources: A.A.A., K.G., W.A., A.S.K., and S.R.; data curation: A.A.A., K.G., A.S.K., W.A., and S.R.; writing—original draft preparation: A.S.K., A.A.A., and W.A.; writing-review and editing: A.A.A., A.S.K., and W.A.; supervision: A.A.A. and K.G.; project administration: A.A.A., K.G., A.S.K., and S.R.; funding acquisition: A.A.A. All authors have read and agreed to the published version of the manuscript. 
Funding: The University of the West Indies, St. Augustine Campus Research and Publication Fund Committee approved the funding for the project (Research grant \#2660-457522 on 3 July 2017). The Tuskegee University, U.S.A. funded the APC.

Institutional Review Board Statement: The study was approved and conducted under terms approved by the University of the West Indies, St. Augustine Campus Research Committee (Research grant \#2660-457522 on 13 April 2016). The UWI St. Augustine Campus Ethics Committee granted the project an exemption from ethical review after assessing the research proposal.

Informed Consent Statement: The investigators obtained the consent of the managers of the four processing plants before they completed the questionnaires (Supplemental data: S2) and before visits were made to the plants to collect samples by the investigators.

Data Availability Statement: All the data are contained within the article and the Supplementary Materials.

Acknowledgments: The technical assistance of Alva Marie Stewart-Johnson and Sannandan Samlal is appreciated. We also thank Joanne Singh and Shayne Ramsubeik for their assistance, as well as the processing plant managers and staff of the four processing plants who participated in the study. We also wish to thank the managers and workers at the four processing plants for facilitating visits to the plants, completion of the questionnaires, and sample collection at the processing plants.

Conflicts of Interest: The authors declare no conflict of interest. The funders had no role in the design of the study; in the collection, analyses, or interpretation of data; in the writing of the manuscript, or in the decision to publish the results.

\section{References}

1. Ferrari, R.G.; Rosario, D.K.A.; Cunha-Neto, A.; Mano, S.B.; Figueiredo, E.E.S.; Conte-Junior, C.A. Worldwide epidemiology of salmonella serovars in animal-based foods: A meta-analysis. Appl. Environ. Microbiol. 2019, 85, e00591-19. [CrossRef]

2. James, S.L.; Abate, D.; Abate, K.H.; Abay, S.M.; Abbafati, C.; Abbasi, N.; Abbastabar, H.; Abd-Allah, F.; Abdela, J.; Abdelalim, A.; et al. GBD 2017 disease and injury incidence and prevalence collaborators. global, regional, and national incidence, prevalence, and years lived with disability for 354 diseases and injuries for 195 countries and territories, 1990-2017: A systematic analy-sis for the global burden of disease study 2017. Lancet 2018, 392, 1789-1858. [CrossRef]

3. GBD Causes of Death Collaborators. Global, regional, and national age-sex-specific mortality for 282 causes of death in 195 countries and territories, 1980-2017: A systematic analysis for the global burden of disease study 2017. Lancet 2018, 392, 1736-1788. [CrossRef]

4. Caribbean Public Health Agency. State of Public Health in the Caribbean Report 2017-2018 Climate and Health: Averting and Responding to an Unfolding Health Crisis; Caribbean Public Health Agency: Port of Spain, Trinidad and Tobago, 2018.

5. Velasquez, C.; Macklin, K.; Kumar, S.; Bailey, M.; Ebner, P.; Oliver, H.; Martin-Gonzalez, F.; Singh, M. Prevalence and antimicrobial resistance patterns of salmonella isolated from poultry farms in southeastern United States. Poult. Sci. 2018, 97, 2144-2152. [CrossRef]

6. Foley, S.L.; Nayak, R.; Hanning, I.B.; Johnson, T.J.; Han, J.; Ricke, S.C. Population dynamics of salmonella enterica serotypes in commercial egg and poultry production. Appl. Environ. Microbiol. 2011, 77, 4273-4279. [CrossRef]

7. Central Statistical Office (CSO)_Government of Trinidad and Tobago. Core Statistics. Available online: https://cso.gov.tt/ (accessed on 21 February 2021).

8. Wright, S. Poultry Production in the Caribbean. Available online: https://www.inciner8.com/blog/animal-incineration/poultry/ poultry-production-in-the-caribbean/ (accessed on 21 February 2021).

9. Johnson, R. Checking in on Caribbean Poultry: Insight from an Expert. Available online: https://www.thepoultrysite.com/ news/2018/09/checking-in-on-caribbean-poultry-insight-from-an-expert (accessed on 21 February 2021).

10. Li, Y.; Yang, Q.; Cao, C.; Cui, S.; Wu, Y.; Yang, H.; Xiao, Y.; Yang, B. Prevalence and characteristics of Salmonella isolates recovered from retail raw chickens in Shaanxi province, China. Poult. Sci. 2020, 99, 6031-6044. [CrossRef]

11. Mokgophi, T.; Gcebe, N.; Fasina, F.; Adesiyun, A. Antimicrobial resistance profiles of salmonella isolates on chickens processed and retailed at outlets of the informal market in Gauteng province, South Africa. Pathogens 2021, 10, 273. [CrossRef]

12. Álvarez-Fernández, E.; Alonso-Calleja, C.; García-Fernández, C.; Capita, R. Prevalence and antimicrobial resistance of Salmonella serotypes isolated from poultry in Spain: Comparison between 1993 and 2006. Int. J. Food Microbiol. 2012, 153, 281-287. [CrossRef]

13. Lamas, A.; Fernandez-No, I.C.; Miranda, J.M.; Vazquez, B.; Cepeda, A.; Franco, C.M. Prevalence, molecular characterization and antimicrobial resistance of Salmonella serovars isolated from northwestern Spanish broiler flocks (2011-2015). Poult. Sci. 2016, 95, 2097-2105. [CrossRef]

14. Folk, M.K. Identifying Production Facility Characteristics in Small and Very Small Meat Processing Plants with Reference to FSIS Salmonella Test Results. Ph.D. Thesis, The Ohio State University, Columbus, OH, USA, 2008. 
15. Henry, I.; Granier, S.; Courtillon, C.; Lalande, F.; Chemaly, M.; Salvat, G.; Cardinale, E. Salmonella enterica subsp. enterica isolated from chicken carcasses and environment at slaughter in Reunion Island: Prevalence, genetic characterization and antibiotic susceptibility. Trop. Anim. Health Prod. 2012, 45, 317-326. [CrossRef]

16. Hamidi, A.; Irsigler, H.; Jaeger, D.; Muschaller, A.; Fries, R. Quantification of water as a potential risk factor for cross-contamination with salmonella, campylobacter and listeriain a poultry abattoir. Br. Poult. Sci. 2014, 55, 585-591. [CrossRef] [PubMed]

17. Schambach, B.T.; Berrang, M.E.; Harrison, M.A.; Meinersmann, R.J. Chemical additive to enhance antimicrobial efficacy of chlorine and control cross-contamination during immersion chill of broiler carcasses. J. Food Prot. 2014, 77, 1583-1587. [CrossRef] [PubMed]

18. Wang, H.; Shu, R.; Zhao, Y.; Zhang, Q.; Xu, X.; Zhou, G. Analysis of ERIC-PCR genomic polymorphism of salmonella isolates from chicken slaughter line. Eur. Food Res. Technol. 2014, 239, 543-548. [CrossRef]

19. Cardoso, A.; Tessari, E. Salmonella in food safety. Biológicol 2008, 70, 11-13. (In Portuguese)

20. Brito, D.A.P.; Sousa, G.L.A.; De Souza, Y.L.; Reis, V.; de Sousa Silva, J.R.; Reis, A.; Oba, A. Sources of paratyphoid salmonella in the production chain of broilers in the northern mesoregion of Maranhão state, Brazil. Semin. Ciências Agrárias 2019, 40, 3021-3034. [CrossRef]

21. Xiao, X.; Wang, W.; Zhang, J.; Liao, M.; Yang, H.; Fang, W.; Li, Y. Modeling the reduction and cross-contamination of salmonella in poultry chilling process in China. Microorganisms 2019, 7, 448. [CrossRef]

22. Durso, L.M.; Cook, K.L. Impacts of antibiotic use in agriculture: What are the benefits and risks? Curr. Opin. Microbiol. 2014, 19, 37-44. [CrossRef]

23. Finley, R.L.; Collignon, P.; Larsson, D.G.J.; McEwen, S.A.; Li, X.-Z.; Gaze, W.H.; Reid-Smith, R.; Timinouni, M.; Graham, D.W.; Topp, E. The scourge of antibiotic resistance: The important role of the environment. Clin. Infect. Dis. 2013, 57, 704-710. [CrossRef]

24. Ziech, R.E.; Lampugnani, C.; Perin, A.P.; Sereno, M.J.; Sfaciotte, R.A.P.; Viana, C.; Soares, V.M.; Pinto, J.P.D.A.N.; Bersot, L.D.S Multidrug resistance and ESBL-producing salmonella spp. isolated from broiler processing plants. Braz. J. Microbiol. 2016, 47, 191-195. [CrossRef]

25. Dan, S.D.; Tăbăran, A.; Mihaiu, L.; Mihaiu, M. Antibiotic susceptibility and prevalence of foodborne pathogens in poultry meat in Romania. J. Infect. Dev. Ctries. 2015, 9, 35-41. [CrossRef]

26. Chon, J.-W.; Jung, H.-I.; Kuk, M.; Kim, Y.-J.; Seo, K.-H.; Kim, S.-K. High occurrence of extended-spectrum $\beta$-lactamase-producing salmonellain broiler carcasses from poultry slaughterhouses in south Korea. Foodborne Pathog. Dis. 2015, 12, 190-196. [CrossRef] [PubMed]

27. Brown, A.C.; Grass, J.E.; Richardson, L.C.; Nisler, A.L.; Bicknese, A.S.; Gould, L.H. Antimicrobial resistance in salmonella that caused foodborne disease outbreaks: United States, 2003-2012. Epidemiol. Infect. 2017, 145, 766-774. [CrossRef] [PubMed]

28. Bearson, B.L.; Bearson, S.M.D.; Looft, T.; Cai, G.; Shippy, D.C. Characterization of a multidrug-resistant salmonella enterica serovar heidelberg outbreak strain in commercial Turkeys: Colonization, transmission, and host transcriptional response. Front. Veter. Sci. 2017, 4, 156. [CrossRef] [PubMed]

29. International Organization for Standardization. ISO 6579-1: 2017 Microbiology of the Food Chain. Horizontal Method for the Detection, Enumeration and Serotyping of Salmonella_Part 1: Detection of Salmonella spp.; International Organization for Standardization: Geneva, Switzerland, 2017.

30. US Department of Agriculture Food Safety and Inspection Service. Isolation and Identification of Salmonella from Meat, Poultry, Pasteurized Egg and Siluriformes(Fish) Products and Carcass and Environmental Sponges; U.S Department of Agriculture: Washington, DC, USA, 2013.

31. Jimenez, S.M.; Salsi, M.S.; Tiburzi, M.C.; Pirovani, M.E. A comparison between broiler chicken carcasses with and without visible faecal contamination during the slaughtering process on hazard identification of Salmonella spp. J. Appl. Microbiol. 2002, 93, 593-598. [CrossRef]

32. Jetton, J.P.; Bilgili, S.F.; Conner, D.E.; Kotrola, J.S.; Reiber, M.A. Recovery of salmonellae from chilled broiler carcasses as affected by rinse media and enumeration method. J. Food Prot. 1992, 55, 329-332. [CrossRef]

33. Cason, J.A.; Bailey, J.S.; Stern, N.J.; Whittemore, A.D.; Cox, N.A. Relationship between aerobic bacteria, salmonellae and Campylobacter on broiler carcasses. Poult. Sci. 1997, 76, 1037-1041. [CrossRef]

34. Cox, N.A.; Richardson, L.J.; Cason, J.A.; Buhr, R.J.; Vizzier-Thaxton, Y.; Smith, D.P.; Fedorka-Cray, P.J.; Romanenghi, C.P.; Pereira, L.V.B.; Doyle, M.P. Comparison of neck skin excision and whole carcass rinse sampling methods for microbiological evaluation of broiler carcasses before and after immersion chilling. J. Food Prot. 2010, 73, 976-980. [CrossRef]

35. Bourassa, D.V.; Holmes, J.M.; Cason, J.A.; Cox, N.A.; Rigsby, L.L.; Buhr, R.J. Prevalence and serogroup diversity of salmonella for broiler neck skin, whole carcass rinse, and whole carcass enrichment sampling methodologies following air or immersion chilling. J. Food Prot. 2015, 78, 1938-1944. [CrossRef]

36. Berrang, M.E.; Cox, N.A.; Cosby, D.E.; Frye, J.; Jackson, C.R. Detection of salmonella serotypes by overnight incubation of entire broiler carcass. J. Food Saf. 2017, 37, e12298. [CrossRef]

37. Kumar, N.; Mohan, K.; Georges, K.; Dziva, F.; Adesiyun, A.A. Prevalence, serovars, and antimicrobial resistance of salmonella in cecal samples of chickens slaughtered in pluck shops in Trinidad. J. Food Prot. 2019, 82, 1560-1567. [CrossRef]

38. Khan, A.S.; Georges, K.; Rahaman, S.; Abdela, W.; Adesiyun, A.A. Prevalence and serotypes of Salmonella spp. on chickens sold at retail outlets in Trinidad. PLoS ONE 2018, 13, e0202108. [CrossRef] [PubMed] 
39. Khan, A.S.; Georges, K.; Rahaman, S.; Abdela, W.; Adesiyun, A.A. Antimicrobial resistance of Salmonella isolates recovered from chickens sold at retail outlets in Trinidad. J. Food Prot. 2018, 81, 1880-1889. [CrossRef]

40. Thrusfield, M. Veterinary Epidemiology, 2nd ed.; Blackwell Publishing: Oxford, UK, 1995; p. 339.

41. Cox, N.A.; Blankenship, L.C. Comparison of rinse sampling methods for detection of salmonellae on eviscerated broiler carcasses. J. Food Sci. 1975, 40, 1333-1334. [CrossRef]

42. European Commission. Commission Regulation (EC) No 2073/2005 of 15 November 2005 on Microbial Criteria for Food Stuffs; L 338; European Commission: Brussels, Belgium, 2005; pp. 1-26. ISSN 1725-2555.

43. Cox, N.A.; Buhr, R.J.; Smith, D.P.; Cason, J.A.; Rigsby, L.L.; Bourassa, D.V.; Fedorka-Cray, P.J.; Cosby, D.E. Sampling naturally contaminated broiler carcasses for salmonella by three different methods. J. Food Prot. 2014, 77, 493-495. [CrossRef]

44. Olovo, C.V.; Reward, E.E.; Obi, S.N.; Ike, A.C. Isolation, identification and antibiogram of salmonella from cloacal swabs of free range poultry in Nsukka, Nigeria. J. Adv. Microbiol. 2019, 2019, 1-9. [CrossRef]

45. Public Health England. Detection of Salmonella Species. National Infection Service, Food, Water E Environmental Microbiology Standard Method; Public Health England: London, UK, 2017; p. 13.

46. Andrews, W. Manual of food quality control. 4. Rev. 1. Microbiological analysis. Food and drug administration. FAO Food Nutr. Pap. 1992, 14, 1-338.

47. Grimont, P.A.D.; Weill, F.X. Antigenic Formulae of the Salmonella Serovars; World Health Organization and Institut Pasteur: Paris, France, 2007; pp. 1-166.

48. Eijkelkamp, J.M.; Aarts, H.J.M.; van der Fels-Klerx, H.J. Suitability of rapid detection methods for salmonella in poultry slaughterhouses. Food Anal. Methods 2009, 2, 1-13. [CrossRef]

49. Oliveira, S.; Santos, L.; Schuch, D.; Silva, A.; Salle, C.; Canal, C. Detection and identification of salmonellas from poultry-related samples by PCR. Veter. Microbiol. 2002, 87, 25-35. [CrossRef]

50. Clinical and Laboratory Standards Institute. Performance Standards for Antimicrobial Susceptibility Testing; CLSI supplement M100; Clinical and Laboratory Standards Institute: Wayne, PA, USA, 2017.

51. Jacob, J.J.; Solaimalai, D.; Sethuvel, D.P.M.; Rachel, T.; Jeslin, P.; Anandan, S.; Veeraraghavan, B. A nineteen-year report of serotype and antimicrobial susceptibility of enteric non-typhoidal salmonella from humans in southern India: Changing facades of taxonomy and resistance trend. Gut Pathog. 2020, 12, 49. [CrossRef] [PubMed]

52. Chai, S.J.; Cole, D.; Nisler, A.; Mahon, B.E. Poultry: The most common food in outbreaks with known pathogens, United States, 1998-2012. Epidemiol. Infect. 2016, 145, 316-325. [CrossRef]

53. Lanier, W.A.; Hale, K.R.; Geissler, A.L.; Dewey-Mattia, D. Chicken liver-Associated outbreaks of campylobacteriosis and salmonellosis, united states, 2000-2016: Identifying opportunities for prevention. Foodborne Pathog. Dis. 2018, 15, 726-733. [CrossRef]

54. Bai, L.; Lan, R.; Zhang, X.; Cui, S.; Xu, J.; Guo, Y.; Li, F.; Zhang, D. Prevalence of salmonella isolates from chicken and pig slaughterhouses and emergence of ciprofloxacin and cefotaxime co-resistant S. enterica serovar indiana in Henan, China. PLoS ONE 2015, 10, e0144532. [CrossRef]

55. Borges, K.; Furian, T.; Souza, S.; Salle, C.; Moraes, H.; Nascimento, V. Antimicrobial resistance and molecular characterization of salmonella enterica serotypes isolated from poultry sources in Brazil. Braz. J. Poult. Sci. 2019, 21, 21. [CrossRef]

56. Jamshidi, A.; Bassami, M.R.; Nik, S. Identification of salmonella spp. and salmonella typhimurium by a multiplex PCR-based assay from poultry carcasses in Mashhad-Iran. Int. J. Vet. Res. 2009, 3. [CrossRef]

57. Svobodova, I.; Borilova, G.; Hulankova, R.; Steinhauserova, I. Microbiological quality of broiler carcasses during slaughter processing. Acta. Vet. 2012, 81. [CrossRef]

58. Rivera-Pérez, W.; Barquero-Calvo, E.; Zamora-Sanabria, R. Salmonella contamination risk points in broiler carcasses during slaughter line processing. J. Food Prot. 2014, 77, 2031-2034. [CrossRef]

59. Jamshidi, A.; Naghdipour, D. Contamination of water used for chilling of poultry carcasses to salmonella typhimurium and salmonella enteritidis using multiplex-PCR method. J. Vet. Res. 2011, 66, 149-152.

60. Monte, D.F.M.; Andrigheto, C.; Ribeiro, V.B.; Landgraf, M.; Destro, M.T. Highly clonal relationship among Salmonella Enteritidis isolates in a commercial chicken production chain, Brazil. Braz. J. Microbiol. 2020, 51, 2049-2056. [CrossRef]

61. Lin, C.-H.; Huang, J.-F.; Sun, Y.-F.; Adams, P.J.; Lin, J.-H.; Robertson, I.D. Detection of chicken carcasses contaminated with Salmonella enterica serovar in the abattoir environment of Taiwan. Int. J. Food Microbiol. 2020, 325, 108640. [CrossRef]

62. Dookeran, M.M.; Baccus-Taylor, G.S.; Akingbala, J.O.; Tameru, B.; Lammerding, A.M. Transmission of salmonella on broiler chickens and carcasses from production to retail in trinidad and tobago. J. Agric. Biodivers. Res. 2012, 1, 78-84.

63. Ramírez-Hernández, A.; Varón-García, A.; Sánchez-Plata, M.X. Microbiological profile of three commercial poultry processing plants in Colombia. J. Food Prot. 2017, 80, 1980-1986. [CrossRef]

64. Yang, H.; Wang, S.; Li, Y.; Johnson, M. Predictive models for the survival/death of campylobacter jejuni and salmonella typhimurium in poultry scalding and chilling. J. Food Sci. 2002, 67, 1836-1843. [CrossRef]

65. Mead, G.C.; Hudson, W.R.; Hinton, M.H. Use of a marker organism in poultry processing to identify sites of cross-contamination and evaluate possible control measures. Br. Poult. Sci. 1994, 35, 345-354. [CrossRef]

66. Wu, D.; Alali, W.Q.; Harrison, M.A.; Hofacre, C.L. Prevalence of salmonella in neck skin and bone of chickens. J. Food Prot. 2014, 77, 1193-1197. [CrossRef] 
67. Mezali, L.; Mebkhout, F.; Nouichi, S.; Boudjellaba, S.; Hamdi, T.-M. Serotype diversity and slaughterhouse-level risk factors related to salmonella contamination on poultry carcasses in Algiers. J. Infect. Dev. Ctries. 2019, 13, 384-393. [CrossRef]

68. Marin, C.; Lainez, M. Salmonella detection in feces during broiler rearing and after live transport to the slaughterhouse. Poult. Sci. 2009, 88, 1999-2005. [CrossRef] [PubMed]

69. NidaUllah, H.; Abirami, N.; Shamila-Syuhada, A.K.; Chuah, L.-O.; Nurul, H.; Tan, T.P.; Abidin, F.W.Z.; Rusul, G. Prevalence of Salmonella in poultry processing environments in wet markets in Penang and Perlis, Malaysia. Veter. World 2017, 10, $286-292$. [CrossRef] [PubMed]

70. Joseph, B.; Otta, S.; Karunasagar, I. Biofilm formation by salmonella spp. on food contact surfaces and their sensitivity to sanitizers. Int. J. Food Microbiol. 2001, 64, 367-372. [CrossRef]

71. Youn, S.Y.; Jeong, O.M.; Choi, B.K.; Jung, S.C.; Kang, M.S. Comparison of the antimicrobial and sanitizer resistance of salmonellaisolates from chicken slaughter processes in Korea. J. Food Sci. 2017, 82, 711-717. [CrossRef]

72. Berghaus, R.D.; Thayer, S.G.; Law, B.F.; Mild, R.M.; Hofacre, C.L.; Singer, R.S. Enumeration of salmonella and campylobacter spp. in environmental farm samples and processing plant carcass rinses from commercial broiler chicken flocks. Appl. Environ. Microbiol. 2013, 79, 4106-4114. [CrossRef] [PubMed]

73. Moore, A.; Nannapaneni, R.; Kiess, A.; Sharma, C.S. Evaluation of USDA approved antimicrobials on the reduction of salmonella and campylobacter in ground chicken frames and their effect on meat quality. Poult. Sci. 2017, 96, 2385-2392. [CrossRef] [PubMed]

74. Lee, N.Y.; Park, S.Y.; Kang, I.S.; Ha, S.D. The evaluation of combined chemical and physical treatments on the reduction of resident microorganisms and salmonella typhimurium attached to chicken skin. Poult. Sci. 2014, 93, 208-215. [CrossRef]

75. Simmons, M.; Fletcher, D.L.; Cason, J.A.; Berrang, M.E. Recovery of salmonella from retail broilers by a whole-carcass enrichment procedure. J. Food Prot. 2003, 66, 446-450. [CrossRef]

76. Cox, N.A.; Berrang, M.E.; House, S.L.; Medina, D.; Cook, K.L.; Shariat, N.W. Population analyses reveal preenrichment method and selective enrichment media affect salmonella serovars detected on broiler carcasses. J. Food Prot. 2019, 82, 1688-1696. [CrossRef] [PubMed]

77. Giombelli, A.; Cavani, R.; Gloria, M.B.A. Evaluation of three sampling methods for the microbiological analysis of broiler carcasses after immersion chilling. J. Food Prot. 2013, 76, 1330-1335. [CrossRef] [PubMed]

78. Adesiyun, A.; Webb, L.; Musai, L.; Louison, B.; Joseph, G.; Stewart-Johnson, A.; Samlal, S.; Rodrigo, S. Survey of salmonella contamination in chicken layer farms in three Caribbean countries. J. Food Prot. 2014, 77, 1471-1480. [CrossRef]

79. Adesiyun, A.A.; Ojo, M.O.; Mohammed, K.; Garcia, G. Frequency of isolation of campylobacters and salmonellae from live broilers reared by contract farmers in Trinidad. Bull. Anim. Health Prod. Afr. 1994, 42, 167-172.

80. EFSA-ECDC: European Food Safety Authority and European Centre for Disease Prevention and Control. The European Union summary report on antimicrobial resistance in zoonotic and indicator bacteria from humans, animals and food in $2017 / 2018$. EFSA J. 2020, 18, e6007. [CrossRef]

81. Caribbean Public Health Agency. State of Public Health in the Caribbean Region Inaugural Report; Caribbean Public Health Agency: Port of Spain, Trinidad and Tobago, 2013.

82. Whiley, H.; Ross, K. Salmonella and eggs: From production to plate. Int. J. Environ. Res. Public Health 2015, 12, 2543-2556. [CrossRef]

83. Borges, K.A.; Furian, T.Q.; De Souza, S.N.; Tondo, E.C.; Streck, A.F.; Salle, C.T.P.; Moraes, H.L.D.S.; Nascimento, V.P.D. Spread of a major clone of salmonella enterica serotype enteritidis in poultry and in salmonellosis outbreaks in southern brazil. J. Food Prot. 2016, 80, 158-163. [CrossRef]

84. Su, C.-P.; De Perio, M.A.; Fagan, K.; Smith, M.L.; Salehi, E.; Levine, S.; Gruszynski, K.; Luckhaupt, S.E. Occupational distribution of campylobacteriosis and salmonellosis cases-Maryland, Ohio, and Virginia, 2014. Morb. Mortal. Wkly. Rep. 2017, 66, 850-853. [CrossRef] [PubMed]

85. Thames, H.T.; Sukumaran, A.T. A Review of Salmonella and Campylobacter in broiler meat: Emerging challenges and food safety measures. Foods 2020, 9, 776. [CrossRef]

86. Mellon, M.G.; Benbrook, C.; Benbrook, K.L. Hogging It: Estimates of Antimicrobial Abuse in Livestock; Union of Concerned Scientists: Cambridge, MA, USA, 2001.

87. Masud, A.A.; Rousham, E.K.; Islam, M.A.; Alam, M.-U.; Rahman, M.; Mamun, A.A.; Sarker, S.; Asaduzzaman, M.; Unicomb, L. Drivers of antibiotic use in poultry production in Bangladesh: Dependencies and dynamics of a patron-client relationship. Front. Veter. Sci. 2020, 7. [CrossRef] [PubMed]

88. Xu, J.; Sangthong, R.; McNeil, E.; Tang, R.; Chongsuvivatwong, V. Antibiotic use in chicken farms in northwestern China. Antimicrob. Resist. Infect. Control 2020, 9, 10. [CrossRef] [PubMed]

89. Romero-Barrios, P.; Deckert, A.; Parmley, E.J.; LeClair, D. Antimicrobial resistance profiles of escherichia coli and salmonella isolates in Canadian broiler chickens and their products. Foodborne Pathog. Dis. 2020, 17, 672-678. [CrossRef] 